\title{
RECENT INNOVATIONS IN MUON BEAM COOLING AND PROSPECTS FOR MUON COLLIDERS
}

\author{
Rolland P. Johnson", Mohammad Alsharo'a, Pierrick M. Hanlet, Robert Hartline, \\ Moyses Kuchnir, Kevin Paul, Thomas J. Roberts (Muons, Inc, Batavia), \\ Charles Ankenbrandt, Emanuela Barzi, Licia Del Frate, Ivan Gonin, Alfred Moretti, David Neuffer, \\ Milorad Popovic, Gennady Romanov, Daniele Turrioni, Victor Yarba (Fermilab, Batavia, Illinois), \\ Daniel M. Kaplan, Katsuya Yonehara (Illinois Institute of Technology, Chicago, Illinois), \\ Kevin Beard, S. Alex Bogacz, Yaroslav Derbenev (Jefferson Lab, Newport News, Virginia)
}

\section{Abstract}

New ideas are being developed to cool muon beams for colliders, neutrino factories, and muon experiments. Analytical and simulation studies have confirmed that a six-dimensional (6D) cooling channel based on helical magnets surrounding RF cavities filled with dense hydrogen gas can be used to achieve very small emittances. This helical cooling channel (HCC) has solenoidal, helical dipole, and helical quadrupole magnetic fields to generate emittance exchange and achieve 6D emittance reduction of over 3 orders of magnitude in a $100 \mathrm{~m}$ segment. Three such sequential HCC segments, where the RF frequencies are increased and transverse dimensions reduced as the beams become cooler, implies a $6 \mathrm{D}$ emittance reduction of almost six orders of magnitude. Two new post-cooling ideas then can be employed to reduce transverse emittances to one or two $\mathrm{mm}$-mr, which allows high luminosity with fewer muons than previously imagined. We describe the new post-cooling ideas as well as a new precooling idea based on a HCC with $z$-dependent fields that can be used as an exceptional 6D cooling demonstration experiment.

\section{INTRODUCTION}

The projects discussed below are to improve the cooling of intense muon beams, which could reduce costs of neutrino factories and facilitate designs of highluminosity muon colliders. Taken together, these SBIR and STTR projects represent a coherent program to develop high intensity, low emittance muon beams to be used in these and other projects.

\section{HIGH-PRESSURE RF CAVITIES}

A gaseous energy absorber enables an entirely new technology to generate high accelerating gradients for muons by using the high-pressure region of the Paschen curve [1]. This idea of filling RF cavities with gas is new for particle accelerators and is only possible for muons because they do not scatter as do strongly interacting protons or shower as do less-massive electrons. Measurements by Muons, Inc. and IIT at Fermilab have

\footnotetext{
* Supported by DOE SBIR/STTR grants DE-FG02-02ER86145, 03ER83722, 04ER84015, 04ER86191, and 04ER84016

\# rol@muonsinc.com
}

demonstrated that hydrogen gas suppresses RF breakdown very well, about a factor six better than helium at the same temperature and pressure. Consequently, much more gradient is possible in a hydrogen-filled RF cavity than is needed to overcome the ionization energy loss, provided one can supply the required RF power. Hydrogen is also twice as good as helium in ionization cooling effectiveness, viscosity, and heat capacity. Present research efforts include tests of pressurized RF Cavities in magnetic fields and high radiation environments and the use of new cavity construction materials [2], including beryllium RF windows for improved cavity performance [3].

\section{Combined Capture, Phase Rotation, Cooling}

High-pressure RF cavities near the pion production target can be used to simultaneously capture, bunch rotate, and cool the muon beam as it emerges from the decaying pions [4]. We have started an $\mathrm{R} \& \mathrm{D}$ effort to develop RF cavities that will operate in the extreme conditions near a production target and an effort to simulate the simultaneous capture, phase rotation, and cooling of muons as they are created from pion decay.

\section{HELICAL COOLING CHANNELS}

The idea of pressurized RF cavities led to the concept of a cooling channel filled with a continuous homogeneous absorber. Such a cooling channel provides longitudinal ionization cooling by exploiting the path length (and therefore energy loss) correlation with momentum in a magnetic channel with positive dispersion. Using this approach in a helical cooling channel creates emittance exchange and excellent 6D muon beam cooling [5]. Figure 1 shows a comparison of results of G4BL and ICOOL simulations of a HCC filled with $200 \mathrm{MHz}$ RF cavities pressurized with hydrogen gas to a density corresponding to half the density of liquid hydrogen. The simulations agree with each other and show nearly equal values of the three cooling decrements and a factor of 5000 reduction of $6 \mathrm{D}$ emittance [6]. For a complete cooling channel, there would be three or four 20 $\mathrm{m}$ long segments, each with higher RF frequency, smaller dimensions, and higher magnetic fields as the beam becomes cooler. 

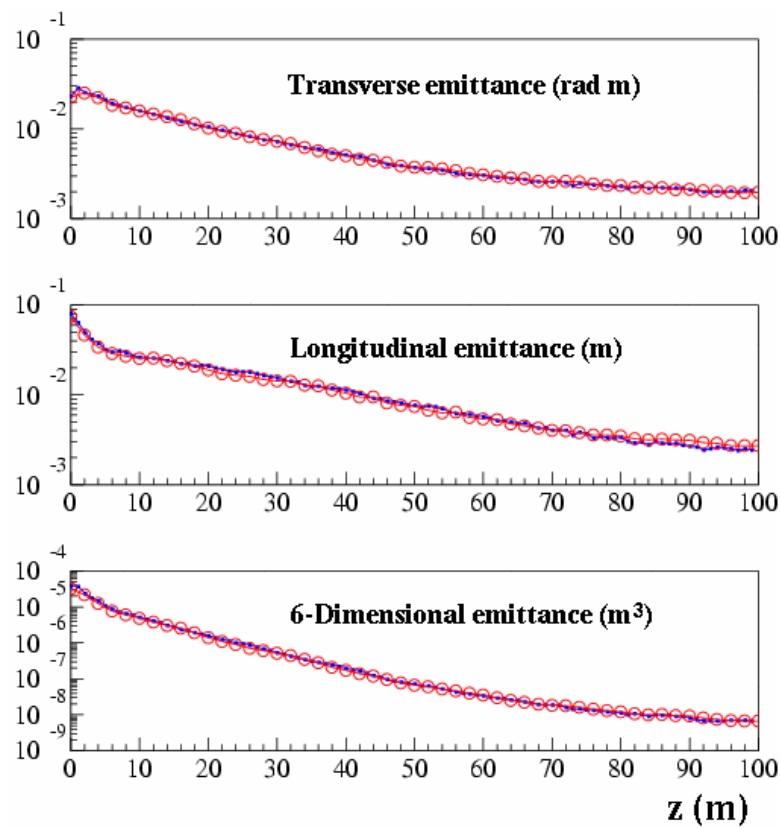

Figure 1: G4BL (Red) and ICOOL (Blue) simulation comparisons of a HCC segment filled with $200 \mathrm{MHz}$ RF cavities pressurized with dense hydrogen gas.

\section{MOMENTUM DEPENDENT HCC}

The HCC concept has now been extended to have momentum-dependent magnetic field strengths for several new applications. Two examples are a 6D precooler which will also serve as a 6D Muon Collider And Neutrino Factory muon beam cooling demonstration experiment, MANX [7], which is being designed to follow MICE [8].

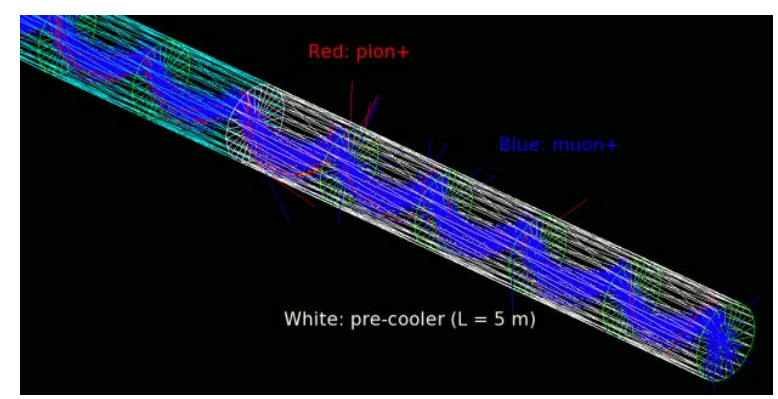

Figure 2: G4BL Openinventor display of a liquid hydrogen filled HCC, without RF, used as a precooler.

\section{Precooler and Sequential RF}

The conceptual change that allowed it to become a $z$ or momentum-dependent device has made the HCC a potential work-horse for different components of the muon beam cooling channel. For example, by filling the HCC with liquid hydrogen or liquid helium, the beam can be decelerated and cooled by ionization energy loss over more than $100 \mathrm{MeV} / \mathrm{c}$, then reaccelerated by a series of RF cavities (pressurized or conventional). The HCC magnet parameters must be varied to match the momentum of the beam as it slows down. Filled with liquid, the HCC with momentum dependent field parameters followed by RF cavities can be a $6 \mathrm{D}$ precooler, a 6D demonstration experiment, or an alternative to the original gas-filled HCC (where the momentum is kept almost constant). Figure 2 shows a G4BL display of a HCC simulation of a $6 \mathrm{~m}$ precooler that follows a $40 \mathrm{~m} \mathrm{HCC}$ decay channel. Filled with gas, the HCC with $z$-dependent magnetic field parameters can be used as a transition between original HCC sections with different RF frequencies and fields.

\section{MANX}

Simulation results in figure 3 show that part of the precooler in figure 2 can serve as the MANX 6D cooling demonstration. The normalized longitudinal emittance is reduced by a factor of 1.7 as are each of the transverse emittances in a HCC segment of $5 \mathrm{~m}$ length. This total 6D emittance reduction of a factor of 5.5 can be compared to the expected MICE cooling effect of about $10 \%$ with no longitudinal cooling. Present studies are to develop a simulation of a MANX design that incorporates the MICE spectrometers and the RAL beamline.
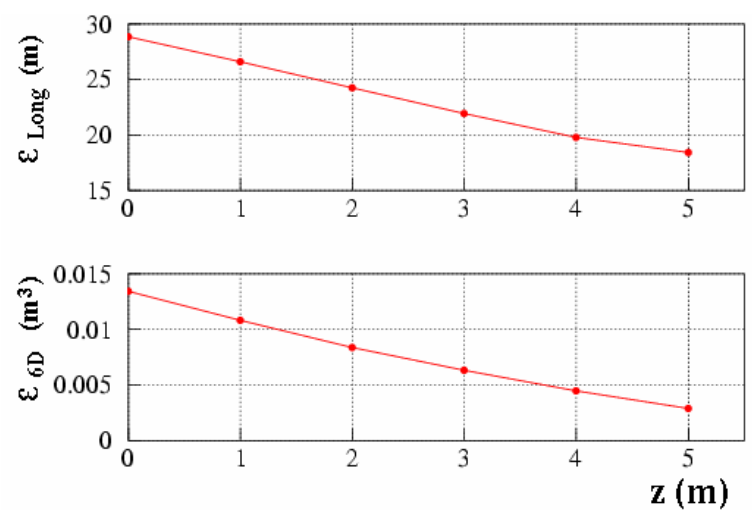

Figure 3: Simulated MANX cooling of (TOP) normalized longitudinal and (BOTTOM) 6D emittances in a $5 \mathrm{~m}$ long HCC filled with liquid hydrogen.

\section{HTS and $\mathrm{H}_{2}$ Cryostats}

Two very interesting technological aspects of HCC designs involve the potential use of HTS to achieve very high fields and the use of hydrogen to act simultaneously as refrigerant, ionization energy absorber, and RF breakdown suppressant [9].

\section{POST COOLING TECHNIQUES}

The excellent cooling that we expect to achieve using the HCC in its various incarnations will bring normalized transverse emittances down to values of a few hundred $\mathrm{mm}-\mathrm{mr}$, which is considerably larger than is usual for electron or proton colliders. Up to now, it has been assumed that a large intensity will make up for this rather large beam size and the emphasis on muon beam technology has been on $4 \mathrm{MW}$ proton drivers and clever production targets. 
The two innovations below, which are now under development, together have the potential to reduce each muon beam normalized transverse emittance another two orders of magnitude to just a few mm-mr, not unlike those presently used in colliders.

\section{Parametric-resonance Ionization Cooling}

Parametric-resonance Ionization Cooling (PIC) [10], requires a half integer resonance to be induced in a ring or beam line such that the normal elliptical motion of particles in $x-x^{\prime}$ phase space becomes hyperbolic, with particles moving to smaller $x$ and larger $x^{\prime}$ as they pass down the beam line. (This is almost identical to the technique used for half integer extraction from a synchrotron where the hyperbolic trajectories go to small $x^{\prime}$ and $\operatorname{larger} x$ to pass the wires of an extraction septum.) Thin absorbers placed at the focal points of the channel then cool the angular divergence of the beam by the usual ionization cooling mechanism where each absorber is followed by RF cavities. Thus in PIC the phase space area is reduced in $x$ due to the dynamics of the parametric resonance and $x^{\prime}$ is reduced or constrained by ionization cooling.

The basic theory of PIC is being developed to include aberrations and higher order effects. Simulations using linear channels of quadrupoles, solenoids, or HCC's are now underway [11].

\section{Reverse Emittance Exchange}

A muon beam that is well cooled at one or two hundred $\mathrm{MeV} / \mathrm{c}$ will have its unnormalized emittance reduced by a factor of a thousand or more at 100 or more $\mathrm{GeV} / \mathrm{c}$ collider energy. At the interaction point in the collider the bunch length would then be much shorter than the IR focal length. In reverse emittance exchange, we propose to repartition the emittances to lengthen each bunch and narrow the transverse emittances using beryllium wedge energy absorbers.

Preliminary calculations show that two stages of reverse emittance exchange, one at low energy and one at a higher energy before energy straggling becomes significant, can reduce each transverse emittance by an order of magnitude.

\section{CONCLUSIONS}

The path to an affordable neutrino factory and a compelling design of a muon collider has complementary projects that we are pursuing with SBIR and STTR grants and proposals, many of which are described in these proceedings.

We are exploring the possible variations of HCC designs, where the original one has shown the promise of several orders of magnitude of $6 \mathrm{D}$ cooling and the new $\mathrm{HCC}$ ideas with momentum dependent magnetic fields are encouraging. The plan for a $6 \mathrm{D}$ cooling experiment using a HCC is taking shape, with a cooling effect that we believe will be striking.
The new post cooling ideas may allow us to have muon beams with transverse emittances as small as those that are now common in the collider world. If this can be accomplished, the number of muons needed in a collider can be reduced by an order of magnitude (for the same luminosity, which is limited by the beam-beam tune shift). The consequences of reduced muon intensity are very significant: the proton driver and targeting requirements can be met with existing machines and technology, the detector backgrounds from muon decay electrons are reduced by a factor of ten, and the site boundary radiation levels due to neutrino interactions in the soil are also reduced by the same factor.

Another important consequence of excellent 6D muon beam cooling is that the cost of acceleration to collider or neutrino factory energy can be reduced by using higher $\mathrm{RF}$ frequency and smaller magnetic channel dimensions. For example, we believe that a one km radius collider ring could be fed by a recirculating muon Linac using $1.3 \mathrm{GHz}$ $\mathrm{RF}$ cavities to create collisions of $5 \mathrm{TeV}$ center of mass energy. Such a machine, which could fit on the Fermilab site, could provide sufficient energy to more than complement the LHC with a total of $5 \mathrm{~km}$ of ILC accelerating sections.

\section{REFERENCES}

[1] R. P. Johnson et al. www.muonsinc.com/TU203.pdf

[2] Pierrick M. Hanlet et al., Studies of RF Breakdown of Metals in Dense Gases, this conference

[3] Mohammad Alsharo'a et al., Beryllium RF Windows for Gaseous Cavities for Muon Acceleration, this conference

[4] Kevin Paul et al., Simultaneous bunching and precooling muon beams with gas-filled RF cavities, this conference

[5] Y. Derbenev and R. P. Johnson, Phys. Rev. ST Accel. Beams 8, 041002 (2005)

[6] Katsuya Yonehara, et al., Simulations of a Gas-Filled Helical Muon Beam Cooling Channel, this conference

[7] Thomas J. Roberts et al., A Muon Cooling Demonstration Experiment Using Gaseous Hydrogen, this conference

[8] M. A. Cummings et al., New Technology in Hydrogen Absorbers, and Michael Green, Progress on the Cryogenic System for a Liquid Absorber in the MICE Cooling Channel, this conference.

[9] Licia Del Frate et al., Novel Muon Cooling Channels Using Hydrogen Refrigeration and HT Superconductor, this conference

[10] Yaroslav Derbenev et al., Ionization Cooling Using a Parametric Resonance, this conference

[11] Kevin Beard et al., Simulations of Parametric Resonance Ionization Cooling of Muon Beams, this conference 